\title{
An ID-Based Key Agreement Protocol Based on ECC Among Users of Separate Networks
}

\author{
Mohammad Sabzinejad Farash \\ Department of mathematics and computer sciences \\ Kharazmi University \\ Tehran, Iran \\ Email: sabzinejad@tmu.ac.ir
}

\author{
Mahmoud Ahmadian Attari \\ Faculty of Electrical and computer Engineering \\ K.N. Toosi University of Technology \\ Tehran, Iran \\ Email: mahmoud@eetd.kntu.ac.ir
}

\begin{abstract}
In this article we propose an identity based key agreement protocol based on elliptic curve cryptography (ECC) between users of different networks with independent private key generations (PKGs). Our protocol is based on Cao et al.'s protocol ,proposed in 2010, in which instead of bilinear pairings, elliptic curves are used for constructing an ID-based key agreement protocol . Our protocol develops Cao et al's protocol for situations that two users of independent organizations or networks with separate servers (that in this article, are named PKGs, because their main duty is generating private keys for the users) want to share a secret key via an insecure link. We also prove the security of the protocol in the random oracle model.
\end{abstract}

Keywords-Identity Based Cryptography, Key Agreement Protocol, Elliptic Curve Cryptography, Random Oracle Model.

\section{INTRODUCTION}

In public key cryptography systems each user has a private key and a corresponding public key. The main problem in this field is how establishing a link between user's identity (ID) and her/his public key. A general solution for this problem is based on Public Key Infrastructure (PKI), defined in ISO/IEC 95948 [1], in which a trust authority, called Certificate Authority (CA), issues a certificate contained user's ID and user's public key signed with his/her private key. Because issuing and using the certificate are costly, another solution named Identity Based Cryptography (IBC) has been proposed.

The IBC idea was first proposed by Shamir in 1984 [2]. In an IBC system, user's ID is considered as her/his public key and the user's private key is generated by a trust authority, called Key Generation Center (KGC) or Private Key Generation (PKG). The main advantage of the IBC systems is that unlike PKI systems, issuing a certificate for each user is not required because there is an inherent link between user's ID and her/his public key.

In 2001, Boneh and Franklin [3] gave the first fully functional solution for ID-based encryption (IBE) using bilinear pairings. Since then, numerous ID-based authenticated key agreement protocols have been proposed based on bilinear pairings (e.g., [4], [5], [6], [7]). In the identity-based cryptosystems, users acquire their private key from the PKG. A single PKG may be responsible for issuing private keys to members of a small-scale organization, but it is unrealistic to assume that a single PKG will be responsible for issuing private keys to members of different organizations, let alone the entire nation or the entire world. Furthermore, it is also unrealistic to assume that different PKGs will share common system parameters and differ only in the master key as done by Chen and Kudla [4]. Therefore, it is needed to consider multiple PKG environment where all the PKGs use different system parameters. In 2005, Lee et al. proposed ID-based 2party and tripartite AK protocol for this setting [8]. However, Kim et al. showed that, these protocol has a serious flaw that allows attackers to impersonate others freely and proposed modifications to the protocol [9].

On performance, according to the results in [10], [11], one bilinear pairing operation requires several times more multiplications in the underlying finite field than an elliptic curve point scalar multiplication does in the same finite field. For lowpower devices such as sensors, cellphones and low-end PDAs, which are usually characterized by limited battery lifetime and low computational power, applications using bilinear pairings can be too expensive to implement. In addition, most of the ID-based cryptosystems require a special hash function called map-to-point hash function [3] for converting a user's identifying information to a point on the underlying elliptic curve. This operation is also time consuming and cannot be treated as a conventional hash operation which is commonly ignored in performance evaluation. To solve the problems which appear due to the bilinear pairings, IBC systems based on elliptic curves have been introduced and developed in various areas including key agreement protocols e.g. [12], [13], [14].

The contribution of this article is to construct a separatePKG type identity based key agreement protocol which does not utilize bilinear pairings, but it uses elliptic curves which is an appropriate choice for low-power and low-memory cryptographic devices. The separate-PKG property allows users of different networks to share a secret key for using in next secure nommunications.

The remainder of the paper is organized as follows: Section 2 focuses on the mathematical background of elliptic curves and security model of the key agreement protocols. In Section 3 we propose an ID-based key agreement protocol without pairing for separate KGCs and prove its security in Section 4. Finally, Section 5 concludes the paper. 


\section{Preliminaries}

\section{A. Elliptic Curve}

An elliptic curve $E$ over a field $\mathbb{F}_{p}$ is defined by an equation

$$
E: y^{2}+a_{1} x y+a_{3} y=x^{3}+a_{2} x^{2}+a_{4} x+a_{6}
$$

where $a_{1}, a_{2}, a_{3}, a_{4}, a_{6} \in F_{p}$ and $\Delta \neq 0$ where $\Delta$ is the discriminate of $E$. The above equation is called the Weierstrass equation. The condition $\Delta \neq 0$ ensures that the elliptic curve is smooth, that is, there are no points at which the curve has two or more distinct tangent lines. Also included in the definition of an elliptic curve is a single element denoted by $\mathcal{O}$ and called the 'point at infinity'. The 'chord and tangent rule' is used for adding two points to give a third point on an elliptic curve. Together with this addition operation, the set of points denoted as $E\left(\mathbb{F}_{p}\right)$ forms a commutative group $\mathbb{G}$ under addition with $\mathcal{O}$ serving as its identity and $P$ as its generation.

Assumption1 Computational Diffie-Hellman (CDH): For $a, b \in \in_{R} \mathbb{Z}_{p}^{*}$, given $P, a P$ and $b P \in \mathbb{G}$, computing $a b P \in \mathbb{G}$ is hard.

\section{B. Security model of key agreement}

We now review the formal security model for ID-based authenticated key agreement protocols due to Chen, Cheng and Smart [15] which is an adapted version of Blake-Wilson et al's model [16]. In the model, each party involved in a session is treated as an oracle, and an adversary can access the oracle by issuing some specified queries (defined later). An oracle $\Pi_{i, j}^{s}$ denotes the $s$-th instance of party $i$ involved with a partner party $j$ in a session. The security of a protocol is defined by a game with two phases. In the first phase, an adversary $E$ is allowed to issue the following queries in any order:

- $\operatorname{Send}\left(\Pi_{i, j}^{s}, x\right)$. Upon receiving the message $x$, oracle $\Pi_{i, j}^{s}$ executes the protocol and responds with an outgoing message $m$ or a decision to indicate accepting or rejecting the session. If the oracle $\Pi_{i, j}^{s}$ does not exist, it will be created as initiator if $x=\lambda$, or as a responder otherwise. In this work, we require $i \neq j$, i.e., a party will not run a session with itself. Such restriction is not unusual in practice.

- $\operatorname{Reveal}\left(\Pi_{i, j}^{s}\right)$. If the oracle has not accepted, it returns $\perp$; otherwise, it reveals the session key. Such an oracle is called opened.

- $\operatorname{Corrupt}(i)$. The party $i$ responds with its private key.

At some point, $E$ can make a Test query to some fresh oracle (Definition 1). E receives either the session key or a random value from a particular oracle.

- $\operatorname{Test}\left(\Pi_{i, j}^{s}\right)$. Oracle $\Pi_{i, j}^{s}$ which is fresh, as a challenger, randomly chooses $b \in\{0,1\}$ and responds with the session key, if $b=0$, or a random sample from the distribution of the session key, otherwise.

In the second phase, the adversary can continue making Send, Reveal and Corrupt queries to the oracles, except that it cannot reveal the test oracle $\Pi_{i, j}^{s}$ or its partner $\Pi_{i, j}^{t}$ (if it exists), and it cannot corrupt party $j$.
- Output: Finally, the adversary outputs a guess $b^{\prime}$ for $b$. If $b^{\prime}=b$, we say that the adversary wins. The adversary's advantage is defined as

$$
A d v^{E}(\kappa)=\left|2 \operatorname{Pr}\left[b^{\prime}=b\right]-1\right|
$$

Definition1 (fresh oracle). An oracle $\Pi_{i, j}^{s}$ is fresh if: 1) $\Pi_{i, j}^{s}$ has accepted; 2$) \Pi_{i, j}^{s}$ is unopened (not been issued the Reveal query); 3) party $j \neq i$ is not corrupted (not been issued the Corrupt query) and 4) there is no opened oracle $\Pi_{i, j}^{t}$, which has had a matching conversation to $\Pi_{i, j}^{s}$.

Definition of security in the model depends on the notion of matching conversations. As mentioned in [15], two oracles have matching conversations to each other if they have the same session identifier which consists of a concatenation of the messages exchanged between them.

A secure authenticated key (AK) agreement protocol is defined as follows:

Definition2. A protocol is a secure AK if:

1) In the presence of a benign adversary, which faithfully conveys messages, on $\Pi_{i, j}^{s}$ and $\Pi_{i, j}^{t}$, both oracles always accept holding the same session key, and this key is distributed uniformly on $0,1^{\kappa}$;

2) For any polynomial time adversary $E, A d v^{E}(\kappa)$ is negligible.

\section{THE PROPOSED PROTOCOL}

An ID-based key agreement protocol between users of separate KGCs is defined in terms of three algorithms system setup, key generation and key agreement.

System Setup: On input $1^{\kappa}$, this algorithm outputs params, a set of system parameters. Basically, this is similar to that of Cao, Kou and Du's work [14]. However, in our system, there are total $n$ different $\mathrm{KGCs,}$ which do not share common system parameters. Therefore, each $\mathrm{KGC}_{i}$ must configure its parameters as follows:

1) Chooses a prime $p^{(i)}$ and determine the tuple $\left\{\mathbb{F}_{p^{(i)}}, E^{(i)} / \mathbb{F}_{p^{(i)}}, G^{(i)}, P^{(i)}\right\}$.

2) Chooses two cryptographic secure hash functions $H_{1}^{(i)}$ : $\{0,1\}^{*} \times \mathbb{G}^{(i)} \longrightarrow \mathbb{Z}_{p^{(i)}}^{*}$.

3) Chooses its master key $x^{(i)} \in R \mathbb{Z}_{p^{(i)}}^{*}$ and compute the system public key $P_{p u b}^{(i)}=x^{(i)} P^{(i)}$.

4) Publishes $\left\{\mathbb{F}_{p^{(i)}}, E^{(i)} / \mathbb{F}_{p^{(i)}}, \mathbb{G}^{(i)}, P^{(i)}, P_{p u b}^{(i)}, H_{1}^{(i)}\right\}$ as system parameters and keep the master key $x^{(i)}$ secret.

Key Generation: Is a key derivation algorithm that on system parameters, master key, and a user's identifier and returns the user's ID-based long-term key. With this algorithm, each $\mathrm{KGC}_{i}$ works as follows for each user $U$ with identifier $I D_{U}$ :

1) Chooses a random $r_{U} \in R \mathbb{Z}_{p^{(i)}}^{*}$, compute $R_{U}=r_{U} P^{(i)}$ and $h_{U}=H_{1}^{(i)}\left(I D_{U}, R_{U}\right)$.

2) Compute $s_{U}=r_{U}+h_{U} x^{(i)}$.

U's private long-term key is $s_{U}$ and is transmitted to $U$ via a secure channel. $U$ can verify his/her private key by checking $s_{U} P^{(i)}=R_{U}+H_{1}^{(i)}\left(I D_{U}, R_{U}\right) P_{p u b}^{(i)}$. 
Key Agreement: Two users $A$ and $B$ of two separate KGCs establish an authenticated secret key as follow:

Step1. $A \rightarrow B:\left\{I D_{A}, T_{A}^{(1)}, T_{A}^{(2)}, R_{A}\right\}$. $A$ chooses two random numbers $a^{(1)} \in_{R} \mathbb{Z}_{p^{(1)}}^{*}$ and $a^{(2)} \in_{R} \mathbb{Z}_{p^{(2)}}^{*}$ and computes $T_{A}^{(1)}=a^{(1)} P^{(1)}$ and $T_{A}^{(2)}=a^{(2)} P^{(2)}$. Then $A$ sends $\left\{I D_{A}, T_{A}^{(1)}, T_{A}^{(2)}, R_{A}\right\}$ to $B$.

Step2. $B \rightarrow A:\left\{I D_{B}, T_{B}^{(1)}, T_{B}^{(2)}, R_{B}\right\} B$ also chooses two random numbers $b^{(1)} \in_{R} \quad \mathbb{Z}_{p^{(1)}}^{*}$ and $b^{(2)} \in_{R} \mathbb{Z}_{p^{(2)}}^{*}$ and computes $T_{B}^{(1)}=a^{(1)} P^{(1)}$ and $T_{B}^{(2)}=a^{(2)} P^{(2)}$. Then $B$ sends $\left\{I D_{B}, T_{B}^{(1)}, T_{B}^{(2)}, R_{B}\right\}$ to $A$.

Step3. Upon receiving the message from $B, A$ computes the shared secrets as follow:

$$
\begin{aligned}
K_{A}^{(1)} & =s_{A} T_{B}^{(1)} \\
K_{A}^{(2)} & =a^{(2)} P_{B}^{(2)}
\end{aligned}
$$

where $P_{B}^{(2)}=s_{B} P^{(2)}=R_{B}+H_{1}^{(2)}\left(I D_{B}, R_{B}\right) P_{p u b}^{(2)}$.

Finally $A$ computes session key with a general one-way hash function like SHA-2 as follows:

$$
\begin{gathered}
K_{A B}=H\left\{I D_{A}, I D_{B}, T_{A}^{(1)}, T_{A}^{(2)}, T_{B}^{(1)}, T_{B}^{(2)}, a^{(1)} T_{B}^{(1)}\right. \\
\left., a^{(2)} T_{B}^{(2)}, K_{A}^{(1)}, K_{A}^{(2)}\right\}
\end{gathered}
$$

Step4. Upon receiving the message from $A, B$ also computes the shared secret keys as follow:

$$
\begin{gathered}
K_{B}^{(1)}=b^{(1)} P_{A}^{(1)} \\
K_{B}^{(2)}=s_{B} T_{A}^{(2)}
\end{gathered}
$$

where $P_{A}^{(1)}=s_{A} P^{(1)}=R_{A}+H_{1}^{(1)}\left(I D_{A}, R_{A}\right) P_{p u b}^{(1)}$.

Finally $B$ also computes the session key with a general one-way hash function like SHA-2 as follows:

$$
\begin{aligned}
& K_{B A}=H\left\{I D_{A}, I D_{B}, T_{A}^{(1)}, T_{A}^{(2)}, T_{B}^{(1)}, T_{B}^{(2)}, b^{(1)} T_{A}^{(1)}\right. \\
&\left., b^{(2)} T_{A}^{(2)}, K_{B}^{(1)}, K_{B}^{(2)}\right\}
\end{aligned}
$$

\section{SeCURity PROOF}

Theorem1. The protocol is a secure AK, provided the $\mathrm{CDH}$ assumption holds and the hash function $H$ is modeled as a random oracle.

Proof. The first condition in Definition 2.2 follows from the assumption that the two oracles follow the protocol and $E$ is benign. In this case, for our protocol it is clear that, both oracles accept holding the same session key because

$$
K_{A}^{(1)}=s_{A} T_{B}^{(1)}=s_{A} b^{(1)} P^{(1)}=b^{(1)} P_{A}^{(1)}=K_{B}^{(1)}
$$

and

$$
K_{B}^{(2)}=s_{B} T_{A}^{(1)}=s_{B} a^{(1)} P^{(1)}=a^{(1)} P_{B}^{(1)}=K_{A}^{(2)}
$$

Thus, the session keys $K_{A B}$ and $K_{B A}$ (Equations 1 and 2, respectively) are equal.

Now we prove that the protocol meets the second condition. For a contradiction, assume that there is an adversary $E$ against our protocol that has a non-negligible advantage $\epsilon$ in guessing correctly whether the response to a Test query is real or random (i.e., winning the attacking game). Apart from this adversary, we show how to construct a simulator $S$ that solves the $\mathrm{CDH}$ problem with non-negligible advantage $\epsilon(\kappa)$. Suppose $\mathrm{A}$ is given an instance $(a P, b P) \in \mathbb{G}$ of the $\mathrm{CDH}$ problem, and is tasked to compute $c P \in \mathbb{G}$ with $c=a b \bmod$ $p$.

We assume that the game between $S$ and $E$ involves $n_{k g c}(\kappa)$ separate KGCs, each KGC can support $n_{u}(\kappa)$ users and each user may be involved in $n_{s}(\kappa)$ sessions where $\kappa$ is the security parameter. As mentioned, our protocol executes between users of separate KGCs so we denote each user $i \in\left\{1, \ldots, n_{u}(\kappa)\right\}$ supported by key generation center $k \in$ $\left\{1, \ldots, n_{k g c}(\kappa)\right\}$ with $i^{(k)}$ and consequently, oracle $\Pi_{i^{(k)} j^{(l)}}^{s}$ denote the $s$-th instance of party $i^{(k)}$ involved with a partner party $j^{(l)}$ in a session. $S$ works by interacting with $E$ as follows:

setup: $S$ simulates the system setup to the adversary $E$ and defines the system public parameters of each KGC. $S$ randomly chooses $\mathcal{K}, \mathcal{L} \in\left\{1, \ldots, n_{\text {pub }}(\kappa)\right\}, I, J \in\left\{1, \ldots, n_{u}(\kappa)\right\}$ (where $I \neq J$ ) and $s \in\left\{1, \ldots, n_{s}(\kappa)\right\}$ and takes the tuple $\left\{\mathbb{F}_{p}, E / \mathbb{F}_{p}, \mathbb{G}, P, P_{p u b}^{(\mathcal{K})}, H_{1}^{(\mathcal{K})}\right\}$ as system public parameters of $\mathrm{KGC}_{\mathcal{K}}$. Then $S$ computes the long-term keys for $U_{i}$ supported by $\mathrm{KGC}_{k}$ denoted by $s_{i}^{(k)} . S$ makes a list $L_{\text {PrivateKeys }}$ whose elements are tuple $\left(I D_{i}^{(k)}, s_{i}^{(k)}\right)$ and determined as follows:

- $\quad$ If $i=I$ and $l=\mathcal{K}$ then take $R_{I}^{(\mathcal{K})}=a P$ which is the input of $\mathrm{CDH}$ problem; hence $S$ does not know the long-term private key $s_{I}^{(\mathcal{K})}$ and inserts $\left(I D_{I}^{(\mathcal{K})}, \perp\right)$ into the list.

Otherwise, $S$ chooses $r_{i}^{(k)} \in Z_{p}^{*}$ at random and computes $R_{i}^{(k)}=r_{i}^{(k)} P^{(k)}, h_{i}^{(k)}=H_{i}^{(k)}\left(I D_{i}^{(k)}, R_{i}^{(k)}\right)$ and private key $s_{i}^{(k)}=r_{i}^{(k)}+r_{i}^{(k)} x^{(k)}$; then $S$ inserts $\left(I D_{i}^{(k)}, s_{i}^{(k)}\right)$ into the list.

Corrupt $\left(\mathbf{I D}_{\mathbf{i}}^{(\mathbf{k})}\right): S$ looks through the list $L_{\text {PrivateKeys. If }}$ $I D_{i}^{(k)}$ is not on the list, $S$ computes the private key and inserts it into the list. $S$ checks the value of $s_{i}^{(k)}$; if $s_{i}^{(k)} \neq \perp$, then $S$ responds it to $E$; otherwise, $S$ aborts the game (Event1).

$\operatorname{Send}\left(\boldsymbol{\Pi}_{\mathbf{i}^{(\mathrm{k})} \mathbf{j}^{(1)}}^{\mathbf{t}}, \mathbf{M}_{\mathbf{1}}, \mathbf{M}_{\mathbf{2}}\right): S$ maintains a list $L_{\text {Send }}$ for each oracle of the form $\left(\Pi_{i^{(k)} j^{(l)}}^{t}, \operatorname{tran}_{i^{(k)} j^{(l)}}^{t},\left(r^{(k)}\right)_{i^{(k)} j^{(l)}}^{t}\right.$, $\left.\left(r^{(l)}\right)_{i^{(k)} j^{(l)}}^{t}, M_{1}, M_{2},\left(K^{(k)}\right)_{i^{(k)} j^{(l)}}^{t},\left(K^{(l)}\right)_{i^{(k)} j^{(l)}}^{t^{(l)}}, S K_{i^{(k)} j^{(l)}}^{t}\right)$ where $\operatorname{tran}_{i^{(k)} j^{(l)}}^{t}$ is the transcript of the oracle so far; $\left(r^{(k)}\right)_{i(k)}^{t} j^{(l)},\left(r^{(l)}\right)_{i^{(k)} j^{(l)}}^{t}$ are random integers used by the oracle to generate messages, $\left(K^{(k)}\right)_{i^{(k)} j^{(l)}}^{t},\left(K^{(l)}\right)_{i^{(k)} j^{(l)}}^{t}$, and $S K_{i^{(k)} j^{(l)}}^{t}$ are set $\perp$ initially. Note that the list $L_{\text {Send }}$ can be updated in other queries as well, such as Reveal and the $H$ queries. $S$ proceeds as follows: 
- $\quad$ If $\Pi_{i^{(k)} j^{(l)}}^{t} \neq \Pi_{I^{(\mathcal{K})} J^{(\mathcal{L})}}^{s}, M$, then $S$ treats according to the protocol.

- Otherwise, $S$ responds with the tuple $\left\{I D_{J}^{(\mathcal{L})}, T_{J}^{(\mathcal{K})}=b P, T_{J}^{(\mathcal{L})}, R_{J}^{(\mathcal{L})}\right\}$ and sets $r_{J}^{(\mathcal{K})}=\perp$ in the list $L_{\text {Send }}$.

$\operatorname{Reveal}\left(\boldsymbol{\Pi}_{\mathbf{i}^{(\mathbf{k})} \mathbf{j}^{(1)}}^{\mathbf{t}}\right): S$ maintains a list $L_{\text {Reveal }}$ with tuples of the form $\left\{\Pi_{i^{(k)} j^{(l)}}^{t}, I D_{i}^{(k)}, I D_{j}^{(l)}, X_{i^{(k)}}^{(1)}, X_{i^{(k)}}^{(2)}, Y_{j^{(l)}}^{(1)}, Y_{j^{(l)}}^{(2)}\right.$, $\left.S K_{i^{(k)}, j^{(l)}}^{t}\right\}$. To respond, first, $S$ looks through to the list $L_{\text {Reveal }}$; if it previously is queried, $S$ responds $S K_{i^{(k)} j^{(l)}}^{t}$ from the list to $E$; otherwise $S$ proceeds in the following way to respond:

- $\quad$ Get the tuple of oracle $\Pi_{i(k) j(l)}^{t}$ from the list $L_{S e n d}$.

- $\quad$ If oracle $\Pi_{i(k)}^{t} j_{(l)}$ has not been accepted, then respond with $\perp$; if $\Pi_{i^{(k)} j^{(l)}}^{t}=\Pi_{I^{(\mathcal{K})} J^{(\mathcal{L})}}^{s}$, then abort the game (Event 2), and if $S K_{i^{(k)} j^{(l)}}^{t} \neq \perp$, return $S K_{i^{(k)} j^{(l)}}^{t}$. Otherwise, look through $L_{H}$;

○ If the tuple $\left\{I D_{i}^{(k)}, I D_{j}^{(l)}, X_{i^{(k)}}^{(1)}, X_{i^{(k)}}^{(2)}\right.$, $\left.Y_{j^{(l)}}^{(1)}, Y_{j^{(l)}}^{(2)}\right\}$ is not in the list, then $S$ selects a random number $S K_{i(k), j(l)}^{t} \in\{0,1\}^{\kappa}$, responds it to $E$ and inserts the tuple $\left\{\Pi_{i^{(k)} j^{(l)}}^{t}, I D_{i}^{(k)}, I D_{j}^{(l)}, X_{i^{(k)}}^{(1)}, X_{i^{(k)}}^{(2)}, Y_{j^{(l)}}^{(1)}\right.$, $\left.Y_{j(l)}^{(2)}, S K_{i(k) j(l)}^{t}\right\}$ into $L_{\text {Reveal }}$;

○ Otherwise (i.e. the tuple $\left\{I D_{i}^{(k)}, I D_{j}^{(l)}, X_{i^{(k)}}^{(1)}, X_{i(k)}^{(2)}, Y_{j^{(l)}}^{(1)}, Y_{j^{(l)}}^{(2)}\right\}$ is in the list $L_{H}$ ), $S$ proceeds as follows:

$\triangleright \quad$ If the existing tuple in $L_{H}$ is of the form $\left\{\Pi_{i^{(k)} j^{(l)}}^{t}, I D_{i}^{(k)}, I D_{j}^{(l)}, X_{i^{(k)}}^{(1)}\right.$, $X_{i^{(k)}}^{(2)}, Y_{j^{(l)}}^{(1)}, Y_{j^{(l)}}^{(2)}, Z_{1 u}, Z_{2 u}, K_{1 u}$, $\left.K_{2 u}, h_{u}\right\}$, then $S$ responds $h_{u}$ to $E$ and updates the list $L_{\text {Reveal }}$.

$\triangleright \quad$ If the existing tuple in $L_{H}$ is of the form $\left\{-, I D_{i}^{(k)}, I D_{j}^{(l)}, X_{i(k)}^{(1)}\right.$, $X_{i(k)}^{(2)}, \quad Y_{j^{(l)}}^{(1)}, Y_{j^{(l)}}^{(2)}, Z_{1 u}, Z_{2 u}, K_{1 u}$, $\left.K_{2 u}, h_{u}\right\}$, then $S$ selects a random number $S K_{i^{(k)} j^{(l)}}^{t} \in\{0,1\}^{\kappa}$, responds it to $E$ and updates the list

$L_{\text {Reveal }}$.

$\triangleright \quad$ If the existing tuple in $L_{H}$ is of the form $\left\{\perp, I D_{i}^{(k)}, I D_{j}^{(l)}\right.$, $X_{i^{(k)}}^{(k)}, \quad X_{i^{(k)}}^{(l)}, \quad Y_{j^{(l)}}^{(k)}, \quad Y_{j^{(l)}}^{(l)}, \quad Z_{1 u}$, $\left.Z_{2 u}, K_{1 u}, K_{2 u}, h_{u}\right\}$, then $S$ checks $Z_{1 u} \in G^{(k)}, Z_{2 u} \in G^{(l)}$, $e\left(X_{i^{(k)}}^{(k)}, Y_{j^{(l)}}^{(k)}\right)=e\left(Z_{1 u}, P^{(k)}\right)$, $e\left(X_{i^{(k)}}^{(l)}, Y_{j^{(l)}}^{(l)}\right)=e\left(Z_{2 u}, P^{(l)}\right)$, $K_{1 u} \in G^{(k)}, K_{2 u} \in G^{(l)}$ and $e\left(P_{i}^{(k)}, Y_{j(l)}^{(k)}\right)=e\left(K_{1 u}, P^{(k)}\right)$, $e\left(P_{j}^{(l)}, X_{j(k)}^{(l)}\right)=e\left(K_{1 u}, P^{(l)}\right)$;

$\diamond \quad$ If those hold, $S$ responds $h_{u}$ to $E$ and replaces the tuple $\left\{\perp, I D_{i}^{(k)}, I D_{j}^{(l)}, X_{i^{(k)}}^{(k)}\right.$,
$X_{i(k)}^{(l)}, Y_{j^{(l)}}^{(k)}, Y_{j^{(l)}}^{(l)}, Z_{1 u}, Z_{2 u}$, $\left.K_{1 u}, K_{2 u}, h_{u}\right\}$ with the tuple $\left\{\Pi_{i^{(k)} j^{(l)}}^{t}, I D_{i}^{(k)}, I D_{j}^{(l)}\right.$, $X_{i^{(k)}}^{(1)}, X_{i^{(k)}}^{(2)}, Y_{j^{(l)}}^{(1)}, Y_{j^{(l)}}^{(2)}, Z_{1 u}$, $\left.Z_{2 u}, K_{1 u}, K_{2 u}, h_{u}\right\}$ in the list $L_{H}$; then $S$ updates the list $L_{\text {Reveal }}$.

$\diamond \quad$ Otherwise, $S$ selects a random number $S K_{i^{(k)} j^{(l)}}^{t} \in\{0,1\}^{\kappa}$ and responds it to $E$; then $S$ updates the list $L_{\text {Reveal }}$ and replaces the tuple $\left\{\perp, I D_{i}^{(k)}, \quad I D_{j}^{(l)}, \quad X_{i(k)}^{(k)}\right.$, $X_{i(k)}^{(l)}, Y_{j^{(l)}}^{(k)}, Y_{j^{(l)}}^{(l)}, Z_{1 u}, Z_{2 u}$, $\left.K_{1 u}, \quad K_{2 u}, h_{u}\right\}$ with the tuple $\left\{-, I D_{i}^{(k)}, \quad I D_{j}^{(l)}\right.$, $X_{i(k)}^{(1)}, X_{i(k)}^{(2)}, Y_{j^{(l)}}^{(1)}, Y_{j^{(l)}}^{(2)}, Z_{1 u}$, $\left.Z_{2 u} K_{1 u}, K_{2 u}, h_{u}\right\}$ in the list $L_{H}$.

$\mathbf{H}\left(\mathrm{ID}_{\mathbf{i}}^{(\mathbf{k})}, \mathbf{I D}_{\mathbf{j}}^{(\mathbf{l})}, \mathbf{X}_{\mathbf{i}^{(k)}}^{(\mathbf{1})}, \mathbf{X}_{\mathbf{i}^{(k)}}^{(\mathbf{2})}, \mathbf{Y}_{\mathbf{j}^{(1)}}^{(1)}, \mathbf{Y}_{\mathbf{j}^{(1)}}^{(\mathbf{2})}, \mathbf{Z}_{1 \mathbf{u}}, \mathbf{Z}_{\mathbf{2 u}}, \mathbf{K}_{\mathbf{1 u}}\right.$, $\left.\mathbf{K}_{\mathbf{2 u}}\right): S$ maintains a list $L_{\text {Reveal }}$ with tuples of the form $\left\{\perp, I D_{i}^{(k)}, I D_{j}^{(l)}, X_{i^{(k)}}^{(k)}, X_{i^{(k)}}^{(l)}, Y_{j^{(l)}}^{(k)}, Y_{j^{(l)}}^{(l)}, Z_{1 u}, Z_{2 u}\right.$, $\left.K_{1 u}, K_{2 u}, h_{u}\right\}$. To respond, $S$ first looks through to the list $L_{H}$; if it previously is queried, $S$ responds $h_{u}$ from the list to $E$; otherwise $S$ Looks through to $L_{\text {Reveal }}$ and proceeds in the following way to respond:

- $\quad$ If the tuple $\left\{I D_{i}^{(k)}, I D_{j}^{(l)}, X_{i^{(k)}}^{(1)}, X_{i^{(k)}}^{(2)}, Y_{j^{(l)}}^{(1)}, Y_{j^{(l)}}^{(2)}\right\}$ is not in the list, then $S$ selects a random number $h_{u} \in\{0,1\}^{\kappa}$, responds it to $E$ and inserts the tuple $\left\{\perp, I D_{i}^{(k)}, I D_{j}^{(l)}, X_{i^{(k)}}^{(1)}, X_{i^{(k)}}^{(2)}, Y_{j^{(l)}}^{(1)}, Y_{j^{(l)}}^{(2)}, Z_{1 u}, Z_{2 u}\right.$, $\left.K_{1 u}, K_{2 u}, h_{u}\right\}$ into $L_{H}$;

- Otherwise, $S$ checks $Z_{1 u} \in G^{(k)}, Z_{2 u} \in G^{(l)}$, $e\left(X_{i^{(k)}}^{(k)}, Y_{j^{(l)}}^{(k)}\right)=e\left(Z_{1 u}, P^{(k)}\right), e\left(X_{i^{(k)}}^{(l)}, Y_{j^{(l)}}^{(l)}\right)=$ $e\left(Z_{2 u}, P^{(l)}\right), K_{1 u} \in G^{(k)}, K_{2 u} \in G^{(l)}$ and $e\left(P_{i}^{(k)}, Y_{j(l)}^{(k)}\right)=e\left(K_{1 u}, P^{(k)}\right), e\left(P_{j}^{(l)}, X_{j^{(k)}}^{(l)}\right)=$ $e\left(K_{1 u}, P^{(l)}\right)$;

○ If those hold, $S$ responds $S K_{i^{(k)} j^{(l)}}^{t}$ to $E$ and inserts the tuple $\left\{\Pi_{i(k)}^{t} j^{(l)}, I D_{i}^{(k)}\right.$, $I D_{j}^{(l)}, X_{i^{(k)}}^{(k)}, X_{i^{(k)}}^{(l)}, Y_{j^{(l)}}^{(k)}, Y_{j^{(l)}}^{(l)}, Z_{1 u}, Z_{2 u}$, $\left.K_{1 u}, K_{2 u}, h_{u}=S K_{i^{(k)} j^{(l)}}^{t}\right\}$ into the list $L_{H}$.

- Otherwise, $S$ selects a random number $h_{u} \in$ $\{0,1\}^{\kappa}$, responds it to $E$ and inserts the tuple $\left\{-, I D_{i}^{(k)}, I D_{j}^{(l)}, X_{i^{(k)}}^{(k)}, X_{i^{(k)}}^{(l)}, Y_{j^{(l)}}^{(k)}\right.$, $\left.Y_{j^{(l)}}^{(l)}, Z_{1 u}, Z_{2 u}, K_{1 u}, K_{2 u}, h_{u}\right\}$ into the list $L_{H}$.

Test $\left(\Pi_{\mathbf{i}^{(\mathbf{k})} \mathbf{j}^{(\mathbf{l})}}^{\mathbf{t}}\right)$ : If $\Pi_{i^{(k)} j^{(l)}}^{t} \neq \Pi_{I^{(\mathcal{K})}, J^{(\mathcal{L})}}^{s}, S$ aborts the game (Event 3). Otherwise, $S$ selects a random number $s k \in$ $\{0,1\}^{\kappa}$ and responds to $E$.

Output: the adversary $E$ outputs the guess $b^{\prime} \in\{0,1\}$. 
Now, the simulator $S$ for solving the $\mathrm{CDH}$ problem proceeds as follows:

The shared secret of the Test oracle $\Pi_{I^{(\mathcal{K})} J^{(\mathcal{L})}}^{s}$ is

$$
\begin{aligned}
\left(K^{(\mathcal{K})}\right)_{I^{(\mathcal{K})} J^{(\mathcal{L})}}^{s} & =s_{I}^{(\mathcal{K})} T_{J}^{(\mathcal{K})}=\left(a+x^{(\mathcal{K})}\right) b P \\
& =a b P+b x^{(\mathcal{K})} P=a b P+x^{(\mathcal{K})} T_{J}^{(\mathcal{K})}
\end{aligned}
$$

it is clear that $S$ can easily compute the part $x^{(\mathcal{K})} T_{J}^{(\mathcal{K})}$ of the shared secret by extracting the master private key of $\mathrm{KGC}_{\mathcal{K}}, x^{(\mathcal{K})}$ from setup phase of the game and finding $T_{J}^{(\mathcal{K})}$

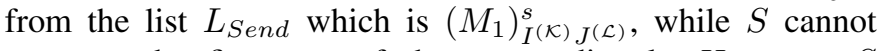
compute the first part of the secret directly. However, $S$ can randomly select a $K_{u}$ from the list $L_{H}$ and compute $Q=K_{u}-x^{(\mathcal{K})}\left(M_{1}\right)_{I^{(\mathcal{K})} J^{(\mathcal{L})}}^{s}$; hence, $Q=a b P$ provided by:

1) Events 1,2 and 3 do not occur;

2) $\left(K^{(\mathcal{K})}\right)_{I^{(\mathcal{K})} J(\mathcal{L})}^{s}$ is in the list $L_{H}$, and

3) $K_{u}=\left(K^{(\mathcal{K})}\right)_{I^{(\mathcal{K})} J^{(\mathcal{L})} \text {. }}^{s}$.

Therefore,

$$
\begin{aligned}
\operatorname{Pr}[Q=a b P]= & \operatorname{Pr}[\overline{\text { Event } 1}, \overline{\text { Event } 2}, \overline{\text { Event } 3}] \\
& \cdot \operatorname{Pr}\left[\left(K^{(\mathcal{K})}\right)_{I^{(\mathcal{K})} J^{(\mathcal{L})}}^{S} \in L_{H}\right] \\
& \cdot \operatorname{Pr}\left[K_{u}=\left(K^{(\mathcal{K})}\right)_{I^{(\mathcal{K})} J^{(\mathcal{L})}}^{s}\right] \\
= & \frac{1}{n_{u}(\kappa) n_{s}(\kappa) n_{p u b}(\kappa)} \operatorname{Pr}\left[\left(K^{(\mathcal{K})}\right)_{I^{(\mathcal{K})} J^{(\mathcal{L})}}^{s} \in L_{H}\right] \\
& \cdot \frac{1}{n_{H}(\kappa)}
\end{aligned}
$$

where $n_{H}(\kappa)$ is number of elements of the list $L_{H}$. Thus, for computing the above probability, $\operatorname{Pr}\left[\left(K^{(\mathcal{K})}\right)_{I^{(\mathcal{K})} J^{(\mathcal{L})}}^{s} \in L_{H}\right]$ should be computed. Assume that the event $A$ is "The adversary wins the game". Therefore, the probability is computed as follows:

$$
\begin{aligned}
& \operatorname{Pr}[A]=\operatorname{Pr}\left[A \mid\left(K^{(\mathcal{K})}\right)_{I^{(\mathcal{K})} J_{J}(\mathcal{L})}^{S} \notin L_{H}\right] \operatorname{Pr}\left[\left(K^{(\mathcal{K})}\right)_{\left.I^{(\mathcal{K})}\right)_{J}(\mathcal{L})}^{S} \notin L_{H}\right]
\end{aligned}
$$

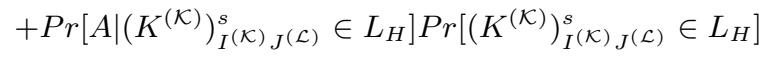

$$
\begin{aligned}
& =\operatorname{Pr}\left[A \mid\left(K^{(\mathcal{K})}\right)_{I^{(\mathcal{K})} J_{J}^{(\mathcal{L})}}^{s} \notin L_{H}\right]\left(1-\operatorname{Pr}\left[\left(K^{(\mathcal{K})}\right)_{\left.I^{(\mathcal{K})}\right)_{J}^{(\mathcal{L})}}^{s} \in L_{H}\right]\right) \\
& +\operatorname{Pr}\left[A \mid\left(K^{(\mathcal{K})}\right)_{I^{(\mathcal{K})} J^{(\mathcal{L})}}^{s} \in L_{H}\right] \operatorname{Pr}\left[\left(K^{(\mathcal{K})}\right)_{I^{(\mathcal{K})} J_{J}^{(\mathcal{L})}}^{S} \in L_{H}\right] \\
& =\operatorname{Pr}\left[A \mid\left(K^{(\mathcal{K})}\right)_{\left.I^{(\mathcal{K})}\right)_{J}(\mathcal{L})}^{s} \notin L_{H}\right]+\left(\operatorname{Pr}\left[\left(K^{(\mathcal{K})}\right)_{\left.I^{(\mathcal{K})}\right)_{J}^{(\mathcal{L})}}^{S} \in L_{H}\right]\right.
\end{aligned}
$$

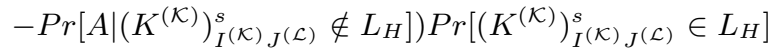

$$
\begin{aligned}
& \leq \frac{1}{2}+\frac{1}{2} \operatorname{Pr}\left[\left(K^{(\mathcal{K})}\right)_{I(\mathcal{K})}^{s}{ }_{J}^{(\mathcal{L})} \in L_{H}\right]
\end{aligned}
$$

Therefore,

$$
\operatorname{Pr}[A] \leq \frac{1}{2}+\frac{1}{2} \operatorname{Pr}\left[\left(K^{(\mathcal{K})}\right)_{I^{(\mathcal{K})} J^{(\mathcal{L})}}^{S} \in L_{H}\right]
$$

On the other hand,

$$
\begin{aligned}
\operatorname{Pr}[A] \geq & \operatorname{Pr}\left[A \mid\left(K^{(\mathcal{K})}\right)_{I^{(\mathcal{K})} J^{(\mathcal{L})}}^{s} \notin L_{H}\right] \\
& \cdot \operatorname{Pr}\left[\left(K^{(\mathcal{K})}\right)_{I^{(\mathcal{K})} J^{(\mathcal{L})}}^{s} \notin L_{H}\right] \\
= & \operatorname{Pr}\left[A \mid\left(K^{(\mathcal{K})}\right)_{I^{(\mathcal{K})} J^{(\mathcal{L})}}^{s} \notin L_{H}\right] \\
& \cdot\left(1-\operatorname{Pr}\left[\left(K^{(\mathcal{K})}\right)_{I^{(\mathcal{K})} J^{(\mathcal{L})}}^{s} \in L_{H}\right]\right) \\
= & \frac{1}{2}-\frac{1}{2} \operatorname{Pr}\left[\left(K^{(\mathcal{K})}\right)_{I^{(\mathcal{K})} J^{(\mathcal{L})}}^{s} \in L_{H}\right]
\end{aligned}
$$

Equations (4) and (5) lead to the following equation:

$$
|2 \operatorname{Pr}[A]-1| \leq \operatorname{Pr}\left[\left(K^{(\mathcal{K})}\right)_{I^{(\mathcal{K})} J^{(\mathcal{L})}}^{s} \in L_{H}\right]
$$

Moreover, we know that

$$
\epsilon(\kappa)=A d v^{E}(\kappa)=|2 \operatorname{Pr}[A]-1|
$$

Then, Equations (6) and (7) follow that

$$
\operatorname{Pr}\left[\left(K^{(\mathcal{K})}\right)_{\left.I^{(\mathcal{K})} J_{(\mathcal{L})} \in L_{H}\right] \geq \epsilon(\kappa)}^{s}\right.
$$

Thus, from Equations (3) and (8), the probability of solving the $\mathrm{CDH}$ problem by the simulator $S$ is as follows:

$$
\begin{aligned}
& \operatorname{Pr}\left[a b P=K_{u}-x^{(K)}\left(M_{1}\right)_{I^{(\mathcal{K})} J^{(\mathcal{L})}}^{s}\right] \\
& \quad=\frac{1}{n_{u}(\kappa) n_{s}(\kappa) n_{p u b}(\kappa) n_{H}(\kappa)} \operatorname{Pr}\left[\left(K^{(\mathcal{K})}\right)_{I^{(\mathcal{K})} J^{(\mathcal{L})}}^{s} \in L_{H}\right] \\
& \geq \epsilon(\kappa) \frac{1}{n_{u}(\kappa) n_{s}(\kappa) n_{p u b}(\kappa) n_{H}(\kappa)}
\end{aligned}
$$

Since the advantage $\epsilon(\kappa)$ is a non-negligible function, the probability of solving the $\mathrm{CDH}$ problem by the simulator $S$ also is non-negligible and this fulfills the proof.

\section{CONCLUSIONS}

In this article we proposed a new identity based key agreement protocol based on elliptic curves between two users supported by separate PKGs that have independent system parameters. Then we proved the security of the protocol in the random oracle model by an extended version of BlakeWilson et al's model. Using elliptic curves causes the protocol be compatible with low-power and lightweight devices, and the property of multi PKGs, enables users of different organizations to share secret keys and establishes a secure communication.

\section{REFERENCES}

[1] ISO/IEC 9594-8:(the 4th edn.) (2001) Information technologyOpen Systems Interconnection-The Directory: Public-key and attribute certificate frameworks. International Organization for Standardization, Geneva, Switzerland.

[2] Shamir, A. (1984) Identity-based cryptosystems and signature schemes, Proc. of CRYPTO1984, LNCS, 196, pp. 47-53.

[3] Boneh, D., Franklin, M., (2001) Identity-based encryption from the weil pairing. Proc. of CRYPTO2001, LNCS, 2139, pp.213229.

[4] Chen, L., Kudla, C., (2003) Identity-based authenticated key agreement protocols from pairing, Proc. of the Computer Security Foundations Workshop, IEEE Press, pp. 219-233. 
[5] Smart, N. (2002) An Identity-based Authenticated Key Agreement Protocol Based on Weil Pairing, Electronic Letters, 38 , 630-632.

[6] McCullagh, N. and Barreto, P. (2005) A new two-party identitybased authenticated key agreement, Proc. of CT-RSA 2005, pp. 262-274.

[7] Yi, X. (2003) Efficient ID-based key agreement from Weil pairing, Electronics Letters, 39, 206-208.

[8] Lee, H., Kim, D., Kim, S. and Oh, H. (2005) Identity-based Key Agreement Protocols in aMultiple PKG Environment. Proc. of the Int. Conf. on Computational Science and Its Applications, ICCSA 2005. Lecture Notes in Computer Science, 3483, 877886, Springer.

[9] Kim, S., Lee, H. and Oh, H. (2005) Enhanced ID-Based Authenticated Key Agreement Protocols for a Multiple Independent PKG Environment, Proc. of ICICS 2005, LNCS, 3783 , 323-335, Springer.

[10] Barreto, P., Kim, H., Lynn, B., and Scott. M. (2002) Efficient algorithms for pairing-based cryptosystems, Proc. CRYPTO 2002, LNCS, 2442 , pp. 354-368, Springer.

[11] Barreto, P., Lynn, B., and Scott. M. (2003) On the selection of pairing-friendly groups. Selected Areas in Cryptography (SAC 2003), LNCS, 3006 , pp. 17-25, Springer.

[12] Cao, X., Kou, W., Yu, Y., Sun, R., (2008) Identity-based authentication key agreement protocols without bilinear pairings, IEICE Trans. Fundam. E91-A, 12, 3833-3836.

[13] Zhu, R.W., Yang, G. and Wong, D.S. (2007) An efficient identitybased key exchange protocol with KGS forward secrecy for lowpower devices, Theor. Comput. Sci., 9 , 198-207.

[14] Cao, X., Kou, W., Yu, Y. and Sun, R. (2010) Identity-based authentication key agreement protocols without bilinear pairings, Information Sciences, 180, 2895-2903.

[15] Chen, L., Cheng, Z. and Smart, N.P. (2007) Identity-based key agreement protocols from pairings, Int. J. Inf. Secur.,6 , 213-241.

[16] Blake-Wilson, S., Johnson, D. and Menezes, A. (1997) Key agreement protocols and their security analysis, Proc. of the 6th IMA International Conference on Cryptography and Coding, pp. $30-45$. 